\title{
SHEAR BOND STRENGTH OF UNIVERSAL-ADHESIVE TO DIFFERENT FIXED PROSTHODONTIC MATERIALS
}

\author{
Diaaeldin Saad Awad*
}

\begin{abstract}
Objectives: To evaluate the shear bond strength (SBS) of Universal adhesive (Single Bond Universal SBU), that contain silane and MDP adhesive molecules, to different fixed prosthodontic materials (High-Leucite ceramics, Zirconia ceramics and base metal alloys).

Methods: Twenty high-leucite based ceramics IPS Empress (Ivoclar) and twenty Ni-cr alloys Remanium-CSe(Dentaurum) specimens were constructed in discs of $5 \mathrm{~mm}$ diameter and $3 \mathrm{~mm}$ thickness. Twenty Zirconia based ceramics INcoris-TZI (Sirona) specimens were prepared of 19X15X3mm blocks. All specimens were embedded into acrylic resin blocks of $2 \times 2 \times 2 \mathrm{~cm}$. Composite discs $3 \mathrm{~mm}$ in diameter Z-350(3M ESPE) were prepared and bonded to each material, divided into 2 groups according to the bonding procedures $(\mathrm{N}=10)$. High-Leucite ceramics etched with 5\% HF acid then for Group 1 composite discs were bonded with Single Bond2 SB2 and RelyX ultimate resin-cement RXU, after Silane $\mathrm{Si}$ application $(\mathrm{Si}+\mathrm{SB} 2+\mathrm{RXU})$, for Group 2 composite discs were bonded with Single Bond Universal adhesive and RelyX Ultimate without separate Silane application (SBU+RXU). Metal and zirconia specimens were air abraded $50 \mu \mathrm{m} \mathrm{AL}_{2} \mathrm{O}_{3}$ then for Group 1; composite discs were bonded with Panavia cement only (Pa). For Group 2, composite discs were bonded with Single Bond Universal and RelyX Ultimate (SBU+RXU). All the specimens were thermo-cycled in water baths $5-55^{\circ} \mathrm{C}$ for 5000 cycles. The SBS was tested in a universal testing machine (Tira) using a chisel-edge blade. The results were statistically analyzed using Mann-Whitney test.
\end{abstract}

Results: bThe mean (SBS \pm SD) in MPa were: In high leucite ceramics (23.1 \pm 2.5$),(23.6 \pm 1.9)$ for (Si+SB2+RUX) and (SBU+RUX) respectively. In Zirconia ceramics: $(7.96 \pm 3.1)$ and (16.8 \pm 5.4) for $(\mathrm{Pa})$ and $(\mathrm{SBU}+\mathrm{RXU})$ respectively. In Base metal alloys: (14.8 \pm 5.4$)$ and $(20.9 \pm 2.7)$ for $(\mathrm{Pa})$ and (SBU+ RXU) respectively. Statistical analysis using Mann-Whitney test showed no significant difference for the high leucite and base metal groups $(P>0.05)$ while there was significant difference for the zirconia groups $(\mathrm{P}<0.05)$.

Conclusion: The Universal-adhesive SBU produced bond strength to high leucite-ceramics comparable to that of resin cement with separate Silane application. It produced higher bond strength to zirconia ceramics and base metal alloys in comparison to Panavia adhesive-resin cement.

KEYWORDS: Universal adhesive, shear bond strength, zirconia-ceramics, leucite-ceramics, base metal alloy.

* Lecturer of crown \& bridge, Department of crown \& bridge, Faculty of Dentistry, Suez Canal University. Ismailia, Egypt 


\section{INTRODUCTION}

The considerable improvements made in adhesive dentistry and dental materials over the last few decades have made non-metallic restorative materials such as ceramic being widely used in daily dental practice to keep up with increasing patient demands for esthetic treatment. Dental ceramics can be broadly defined based on their composition as either silica-based or non-silicabased. This difference has pronounced clinical implications, as hydrofluoric acid treatment only modifies the microstructure of silica-based ceramics (glass ceramics) such as feldspathic porcelain, leucite-reinforced ceramic, and lithium-disilicate ceramic. Non-silica-based ceramics (polycrystalline ceramics) such as alumina and zirconia, on the other hand, are not affected ${ }^{1}$.

Regarding to glass ceramic surface, the conditioning has been purposed with hydrofluoric acid followed by silanation. The acid selectively dissolves the glass matrix creating micromechanical retention, and the silanation serves for the chemical adhesion between the organic and inorganic substances, producing a strong and durable adhesion between the ceramic and the resin cement. ${ }^{2,3}$

Regarding zirconia based ceramics several procedures for adhesive cementation have been investigated. Silica-coating through air abrasion associated to application of silane coupling agents has been largely investigated, ${ }^{4,5,6}$ and resulted in higher bond strength to composite resins compared to other surface treatments, whether in air, water or after thermocycling., ${ }^{45}$ Air abrasion with aluminium oxide roughens the ceramic surface and increases the surface area for bonding, providing mechanical retention. ${ }^{6}$ However, surface damage could lead to immediate clinical consequences or premature failure. ${ }^{7,8}$ For small sized-particles (35$50 \mu \mathrm{m})$ Scherrer et al. ${ }^{9}$ showed improvements in mechanical strength of air abraded yttria stabilized polycrystalline tetragonal zirconia (Y-TZP) specimens after mechanical ageing in humid environment. Primers play an important role in adhesive procedures, especially for zirconia-based ceramics, since the untreated surface of zirconia is not readily reactive to conventional silane agents. An important component that may be present in primers is the 10-methacryloxydecyl dihydrogen phosphate monomer (MDP). This molecule was firstly added to resin cements (e.g. Panavia F, Kuraray, Japan) for bonding to crystalline ceramics having low glass content, resulting in improved adhesion. ${ }^{6,10,11}$ Organo-phosphate monomers contain polymerizable functional groups (e.g. methacrylates), which can polymerize with the matrix of methacrylate-based dental resin cements, composites and adhesives. ${ }^{12}$ The formation of a covalent bond between the oxygen, phosphorus and zirconia $(\mathrm{P}-\mathrm{O}-\mathrm{Zr})$ was confirmed in a study by Chen L. etal. ${ }^{13}$

One of the most recent novelties in adhesive dentistry was the introduction of 'universal' or 'multi-mode' adhesives. These materials are simplified adhesives, usually containing all bonding components in a single bottle. In addition, some universal adhesives may contain silane in their formulation, potentially eliminating the silanization step when bonding to glass ceramics or resin composites. Also it may incorporate MDP molecules that make them suitable for bonding to zirconia based ceramics and base metal alloys. However, the performance of such adhesives is still under investigations. So the purpose of this study was to evaluate the shear bond strength of one of the universal adhesives that contain silane and MDP adhesive molecules (Single bond universal) to different materials used in fixed prosthodontics e.g. glass ceramics, zirconia ceramics and base metal alloys.

The hypothesis tested was that the universal adhesive would have similar bond strength results to the conventional adhesive irrespective of the substrate tested. 


\section{METHODS}

\section{Specimen Preparation}

\section{High leucite ceramics}

Twenty high leucite ceramics (IPS Empress, Ivoclar Vivadent; Schaan, Liechtenstein) discs were constructed using the press technique. $5 \mathrm{~mm}$ in diameter and $3 \mathrm{~m}$ thickness wax discs were constructed using split Teflon mold, sprued, invested and pressed into ceramic discs according to the manufacturer instructions (Programat EP5010, Ivoclar Vivadent; Schaan, Liechtenstein). The ceramic discs were embedded into self-cure acrylic resin blocks $2 \times 2 \times 2 \mathrm{~cm}$ (Acrostone, Egypt). Immediately before bonding all the ceramic discs were etched with 5\% HF for 60 seconds (IPS Ceramic etching Gel, Ivoclar Vivadent; Schaan, Liechtenstein). All specimens were ultrasonically cleaned in distilled water for $15 \mathrm{~min}$ (BlackstoneNey ultrasonic; NY, USA ).

\section{Zirconia}

Twenty zirconia blocks 19X15X3 mm were cut from zirconia ceramic blocks (In-coris TZIblank Sirona Dental Systems GmbH, Bensheim, Germany). The blocks were cut using low speed diamond saw (Micracut 125, Metkon, Bursa, Turkey), cleaned and sintered at $1510^{\circ} \mathrm{C}$ for 120 min (Infire HTC Sirona Dental Systems GmbH, Bensheim, Germany). The zirconia specimens were polished with 600 grit rotating silicon carbid paper and were airborne-particle abraded with $50 \mu \mathrm{m}$ $\mathrm{Al}_{2} \mathrm{O}_{3}$ at 2.5 bars of pressure $(20 \mathrm{~s})$. All specimens were ultrasonically cleaned in distilled water for $15 \mathrm{~min}$ immediately before the bonding procedure.

\section{Base metal alloys}

Twenty Ni-cr alloys Remanium-CSe (Dentaurum) discs were constructed using the lost wax technique. Wax discs of $5 \mathrm{~mm}$ diameter and

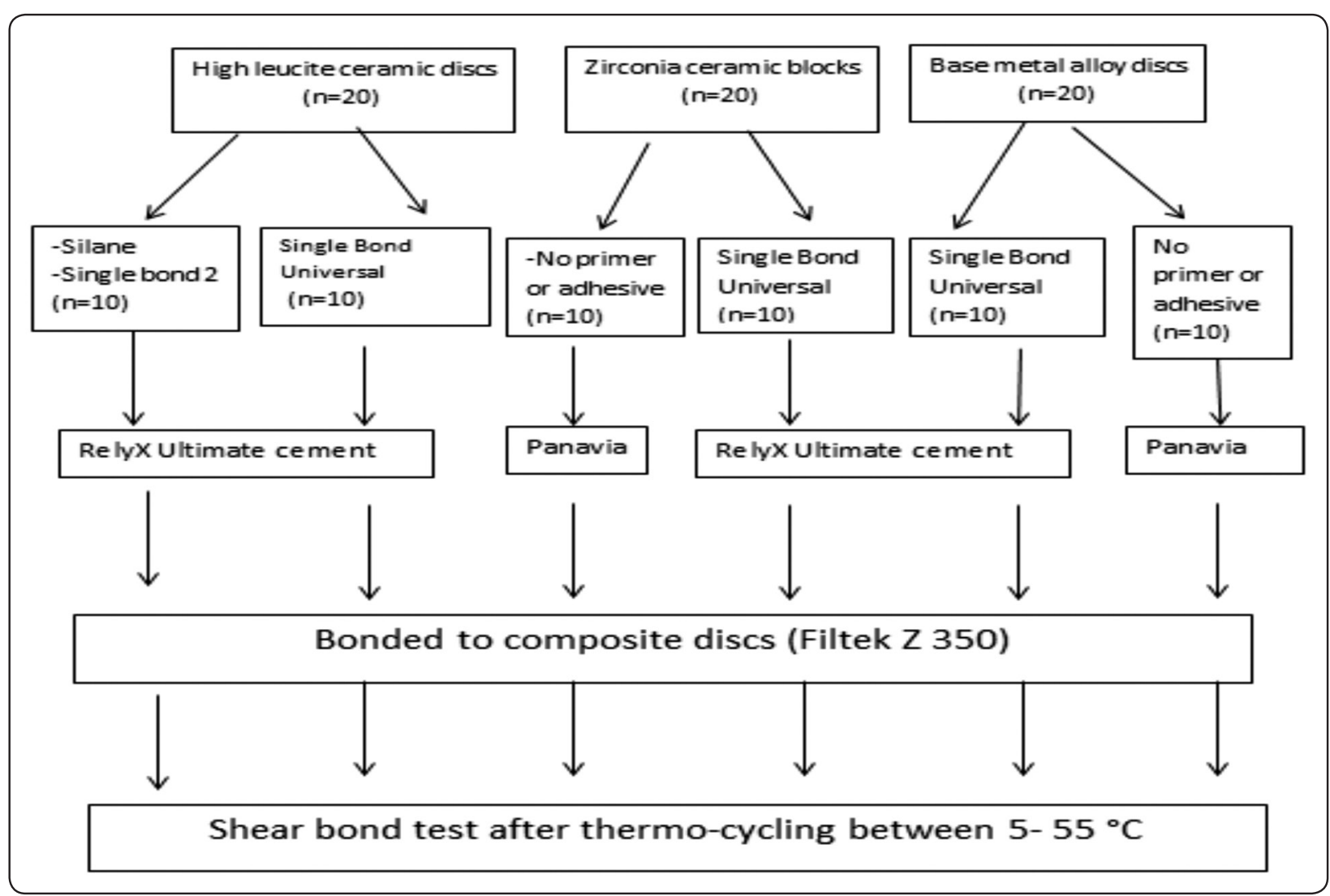

Fig. (1) Experimental design of the study 
$3 \mathrm{~mm}$ thickness were constructed using split Teflon mold, sprued, invested and induction casted into metal discs according to manufacturer instructions. (Fornax, Bego, Bremen, Germenay). The metal discs were airborne- particle abraded with $50 \mu \mathrm{m}$ $\mathrm{AL}_{2} \mathrm{O}_{3}$ at 2.5 bars of pressure $(20 \mathrm{~s})$. All specimens were ultrasonically cleaned in distilled water for $15 \mathrm{~min}$ immediately before the bonding procedure.

\section{Bonding procedures}

Composite discs (Z 350 XT, 3M ESPE, ST Paul, USA) $3 \mathrm{~mm}$ in diameter were constructed using split Teflon mold with $3 \mathrm{~mm}$ inner diameter. Composite discs were bonded to each tested substrate and divided into two subgroups $(\mathrm{n}=10)$ according to the used adhesive either universal adhesive, Single bond Universal, or conventional adhesive, Single bond 2. (Fig.1). A specially constructed device was used during bonding of the samples that allowed application of standardized $3 \mathrm{~kg}$ load. (Fig. 2)

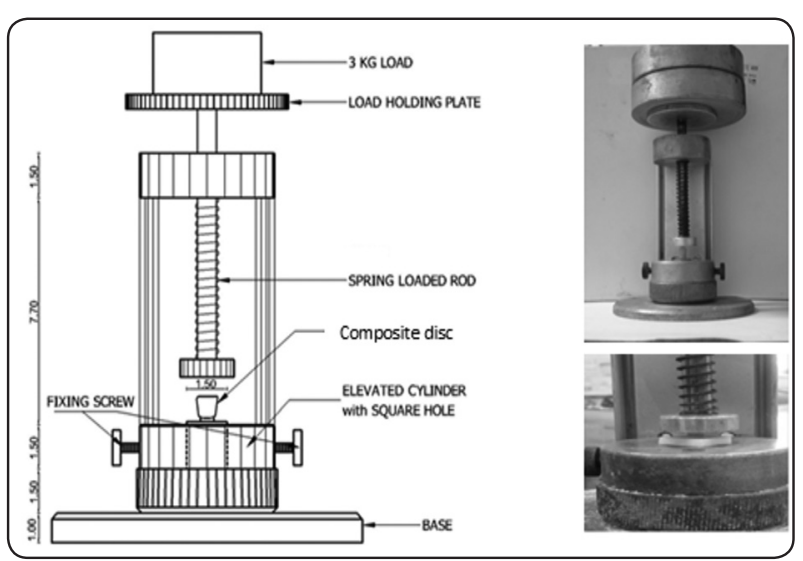

Fig. (2) Specially designed device used during bonding of samples:

TABLE (1): Results of the shear bond strength

\section{Aging, shear bond testing \& analyzing of data}

All the specimens were thermo-cycled in water baths (MPM Instruments, Bernareggio MI, Italy.) between $5-55^{\circ} \mathrm{C}$ for 5000 cycles before the shear test using custom made thermos-cycling machine. The shear bond strength was tested using a universal testing machine (Tira GmbH, Schalkau, Germany). Chisel edge blade was used with cross head speed of $0.5 \mathrm{~mm} / \mathrm{min}$. The shear load necessary to debond the specimen was recorded in Newton $(\mathrm{N})$. The shear bond values in Mega Pascal (MPa) were calculated for all tested groups using the following equation:

Shear bond strength in MPa $=$ Breaking load in $\mathrm{N} /$ bonded surface area in $\mathrm{mm}^{2}$

Bonded area in $\mathrm{mm}^{2}=\pi \mathrm{r}^{2}=3.14 \mathrm{x}(1.5)^{2}=3.14 \mathrm{x}$ $2.25=7.1$

So the value in $\mathrm{MPa}=\operatorname{load} \mathrm{N} / 7.1$

The data were analyzed using Mann-Whitney tests. The overall significance level was set at $(\mathrm{P}$-value $=0.05)$.

\section{RESULTS}

The shear bond strength results are shown in table 1 and presented in figure 3 . For the high leucite ceramic groups no significant difference was found when bonding was done using either the universal adhesive SBU or the conventional adhesive SB2, with separate silane application, (P-value $>0.05$ ). For the zirconia ceramics the universal adhesive produced significantly higher shear bonded strength than when Panavia cement was used (P-value $<0.05)$. For the base metal alloy groups the universal adhesive produced higher bond strength

\begin{tabular}{|c|c|c|c|c|c|c|c|}
\hline & & \multicolumn{2}{|c|}{ G. ceramic } & \multicolumn{2}{|c|}{ Zirconia } & \multicolumn{2}{|c|}{ B. Metal } \\
\hline \multicolumn{2}{|c|}{ Adhesive and cement } & $\begin{array}{c}\text { Silane +SB2+ } \\
\text { RelyX Ultimate }\end{array}$ & $\begin{array}{c}\text { SBU + Rely } \\
\text { X ultimate }\end{array}$ & Panavia & $\begin{array}{c}\text { SBU + Rely } \\
\text { X ultimate }\end{array}$ & Panavia & $\begin{array}{c}\text { SBU + Rely } \\
\text { X ultimate }\end{array}$ \\
\hline \multirow{2}{*}{$\begin{array}{l}\text { Shear bond } \\
\text { strength }\end{array}$} & Mean \pm SD & $23.1 \pm 2.5$ & $23.6 \pm 1.9$ & $7.96 \pm 3.1$ & $16.8 \pm 5.4$ & $14.8 \pm 5.4$ & $20.9 \pm 2.7$ \\
\hline & Range & $19.6-25.7$ & $21.3-25.4$ & $5.11-12.06$ & $9.32-23.08$ & $8.2-20.61$ & $17.47-23.9$ \\
\hline \multicolumn{2}{|l|}{ p-value } & \multicolumn{2}{|c|}{0.7 (NS) } & \multicolumn{2}{|c|}{$0.01 *$} & \multicolumn{2}{|c|}{0.05 (NS) } \\
\hline
\end{tabular}

(NS) Non-significant * Significant 


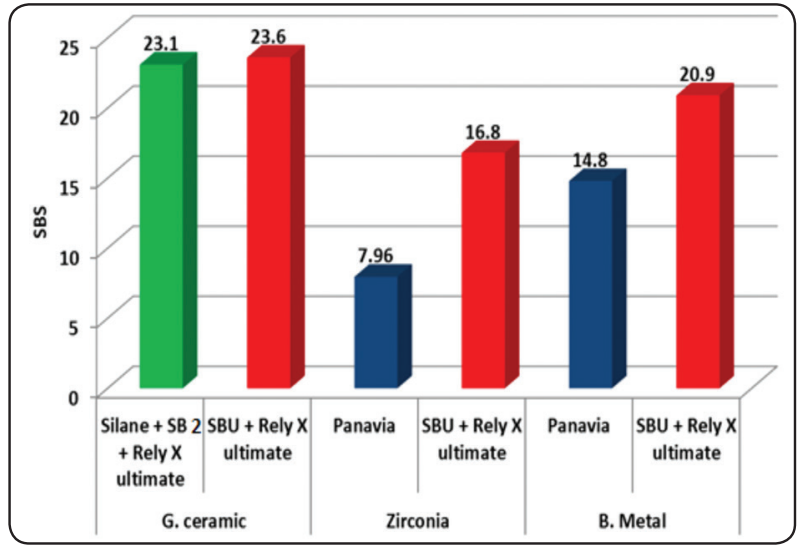

Fig. (3) Shear bond strength among the groups than Panavia cement however the difference was non-significant (P-value $=0.05)$.

Examination of the fractured interface under Stereo-microscope (Nikon MA 100 Japan, Omnimet software) magnification of $10 \mathrm{X}$ showed that for the high leucite ceramic all of the failure modes were cohesive within the ceramics denoting that the bond strength exceeding the fracture strength of the ceramics in both groups (Figure 4). For the zirconia ceramics the failure modes were completely adhesive between resin and zirconia (Figure 5). For the base metal alloy groups the failure modes was predominantly adhesive between resin and base metal about $90 \%$ of the failure patterns and $10 \%$ were mixed with adhesive debonding predominantly with minimal resin residues (Figure 6).

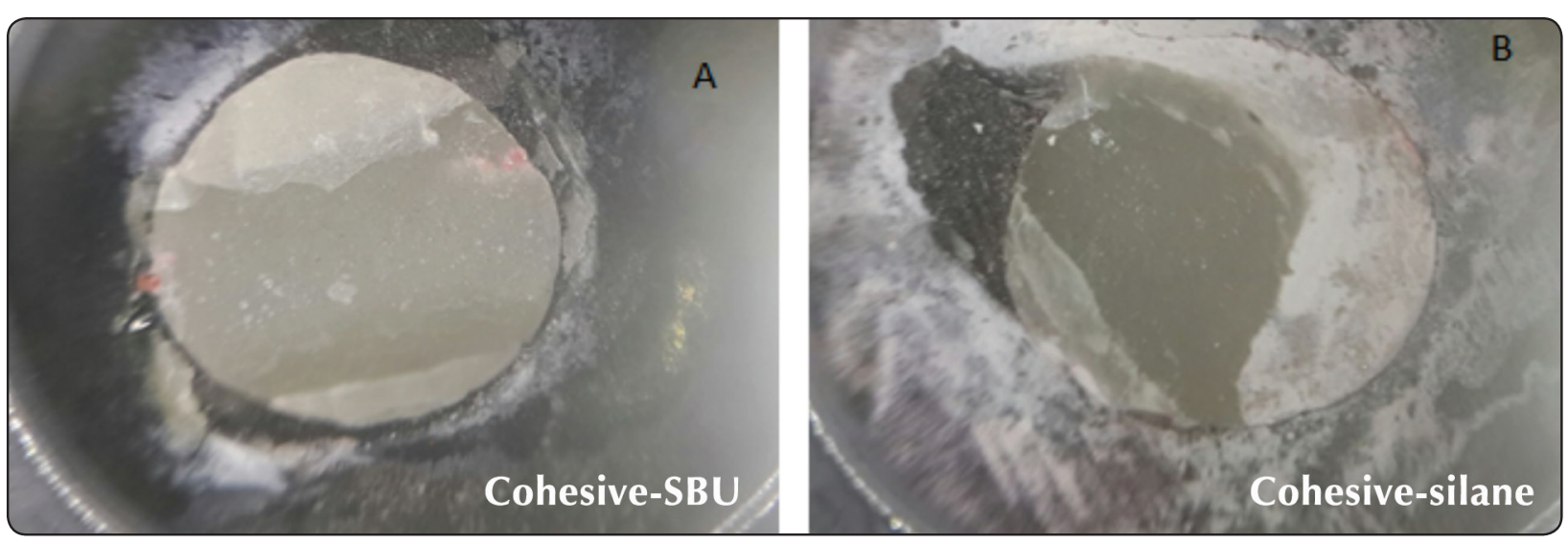

Fig. (4) Cohesive failure within ceramic A: Single bond Universal, B: silane \& Single bond 2

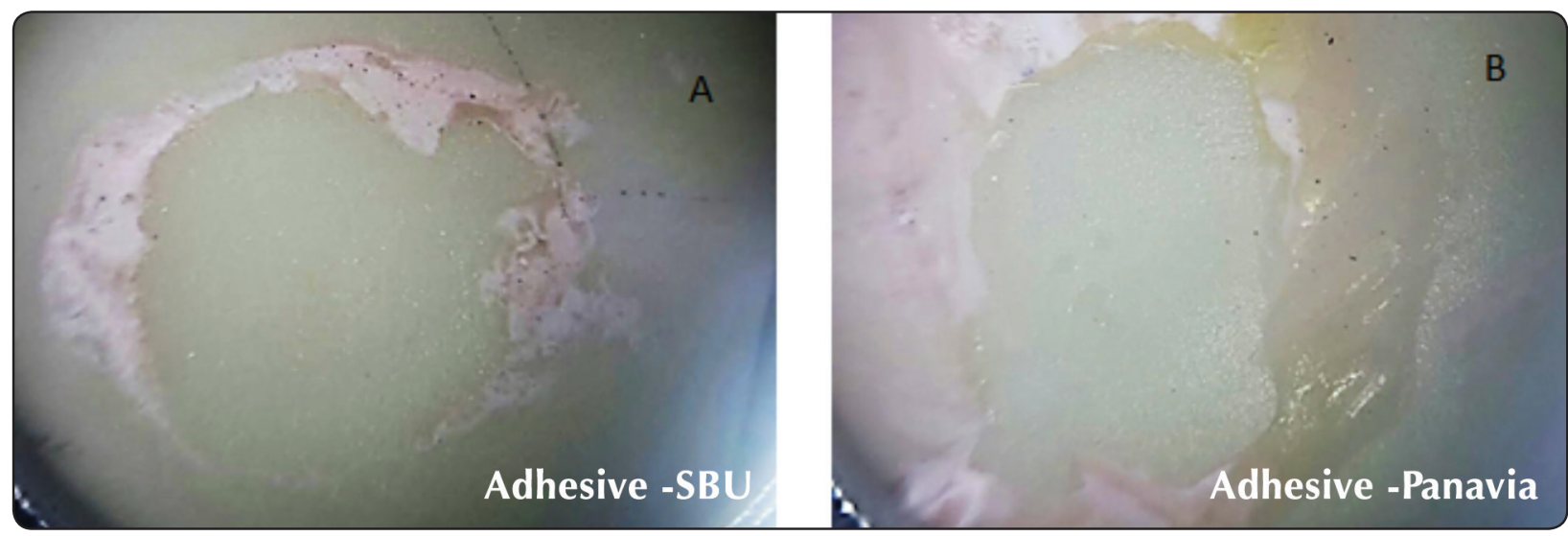

Fig. (5) Adhesive failure between cement and zirconia A: Single bond Universal, B: Panavia 

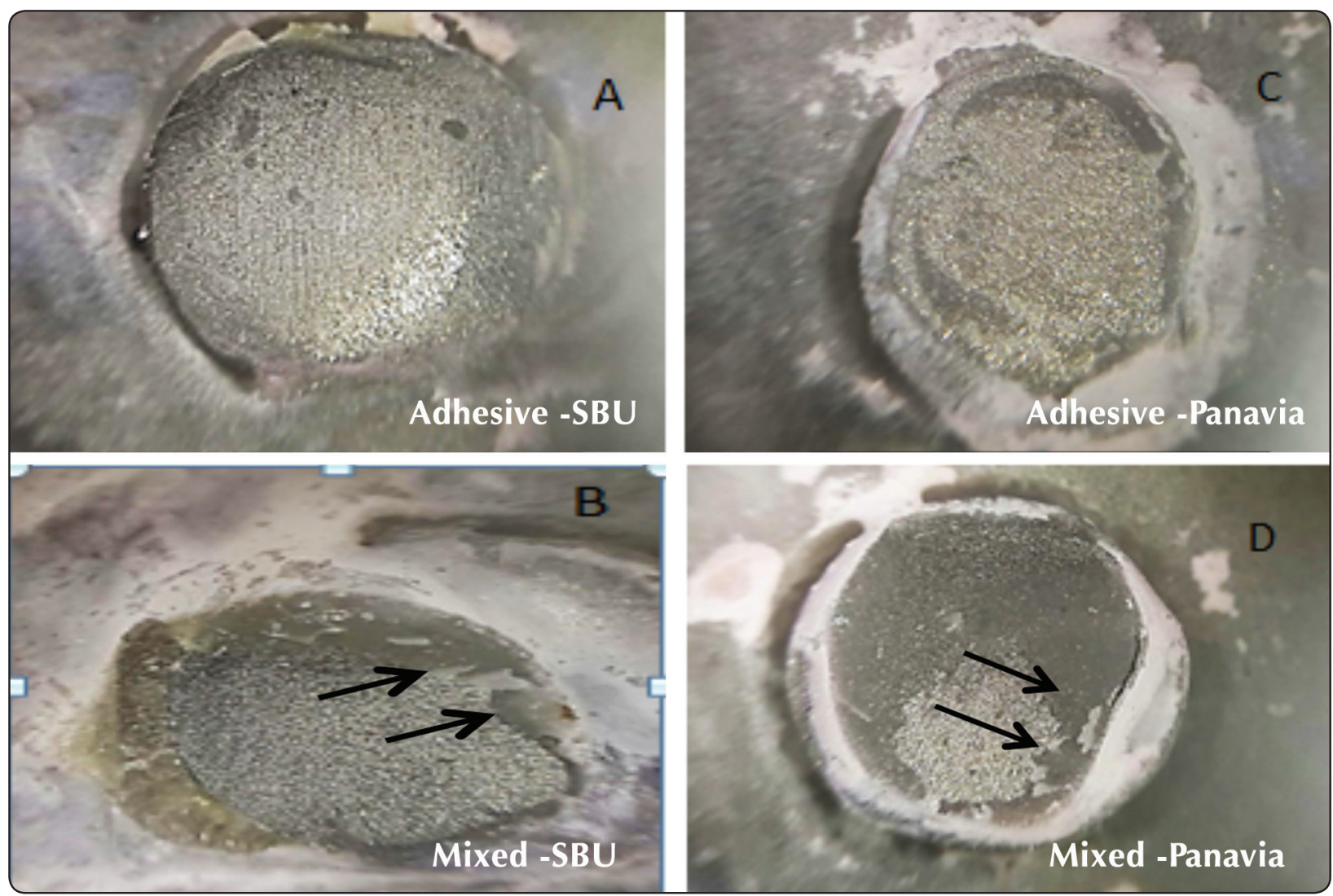

Fig. (6) A\&B Adhesive and mixed failure between cement \&metal (respectively) for Single bond Universal, C\&D, adhesive and mixed failure between cement \&metal (respectively) for Panavia. (Arrows show traces of the resin)

\section{DISCUSSION}

The type of substrate is one of the most important factors affecting the bonding performance of adhesives in dentistry ${ }^{14}$. The chemistry of the substrates that might be dental tissues or restorative materials, may request the application of specific materials to allow a satisfactory and long-lasting bonding. Restorative materials such as resin composites, ceramics, and metals have a low reactive structure after curing/sintering, or casting thus requiring the application of specific components to make their surface active again and prone to adhesion ${ }^{15}$. Some universal adhesives present a versatile formulation that may enable adhesion to any type of substrate, although the performance of universal adhesives tested to different substrates still needs further investigation.
The tested hypothesis was accepted for the high leucite (no difference in bond strength) and rejected for the zirconia and base metal alloy groups as the universal adhesive SBU produced significantly higher shear bond strength to zirconia than panavia cement).

In high leucite ceramic group, SBU showed bond strength similar to the bond strength of the positive control (i.e., application of silane and adhesive). This finding is likely a result of the silane molecule presented in SBU formulation, allowing proper chemical interaction with the glass phases of the ceramic. The results of this study corroborate another study ${ }^{15}$ which showed that SBU produced higher bond strength, than conventional adhesive SB2 and separate silane, to feldspathic porcelain. On the other hand the results come in contrast 
to other study by Kim et $\mathrm{al}^{16}$ who reported lower bond strength of SBU to high leucite ceramics in comparison to positive control. He reported that the acidic functional monomer 10-MDP (methacryloyloxydecyl dihydrogen phosphate) that is incorporated in universal adhesives may impede the ideal chemical interaction between silane and ceramics owing to the tendency for premature hydrolysis in an acidic environment.

For the zirconia and base metal groups single bond universal showed higher bond strength than panavia group. The presence of phosphate monomers in the adhesive allows the formation of covalent bonding to the zirconia surface and the copolymerization to methacrylate groups in resin cements by photo-activation. ${ }^{12,13}$ The interaction between roughened surfaces after air abrasion with $\mathrm{Al}_{2} \mathrm{O}_{3} 50 \mu \mathrm{m}$ particles and adhesive improved adhesion between zirconia and resin cement. These results may be due to an increase in roughness and/ or chemical interaction between $\mathrm{Al}_{2} \mathrm{O}_{3}$ particles and the adhesive, since it contains MDP, which has affinity to metallic oxides. The use of smaller $\mathrm{Al}_{2} \mathrm{O}_{3}$ particles $50 \mu \mathrm{m}$ in this study was to avoid the damaging effect of larger particles $110 \mu \mathrm{m}$. ${ }^{7-9}$ Although both the universal adhesive SBU and the adhesive resin cement Panavia contain MDP molecule, SBU produced higher bond strength than Panavia. This could be explained by the fact that the MDP molecule in SBU is incorporated in a low viscosity adhesive in contrast to Panavia cement which has higher viscosity. So the MDP incorporated in the Universal adhesive SBU is able to wet and diffuse more easily into the zirconia and metal surfaces and subsequently produce higher bond strength. The results of this study come in agreement with another study ${ }^{17}$ by Amaral et al who founded better bond performance after air abrasion with $\mathrm{Al}_{2} \mathrm{O}_{3}$ particles and use of universal primers on zirconia substrates.

\section{Clinical implication}

The advantages of using a universal adhesive, suitable for a wide range of restorative materials, mighteliminate the risk of inappropriate conditioning of the bonding surfaces, simplify application steps, and increase the acceptance of adhesive luting methods in the dental practice. ${ }^{18}$ Furthermore, those adhesives have been shown a good alternative in terms of bond strength to substrate specific primers. ${ }^{18}$ Another good indication for universal adhesives is the use for intraoral repairs, since one may not know the exact nature of the fractured substrate and the use of a universal adhesive will represent a practical alternative.

\section{CONCLUSION}

Universal adhesives containing MDP and Silane appear to be a good option for bonding to Glass ceramics, zirconia, and base metals. Universal adhesives seem to have potential applicability in different areas of the adhesive dentistry.

\section{REFERENCES}

1 Kramer N, Frankenberger R. Clinical performance of bonded leucite-reinforced glass ceramic inlays and onlays after eight years. Dent Mater. 2005;21:262-271.

2 Fabianelli A, Pollington S, Papacchini F, Goracci C, Cantoro A, Ferrari M. The effect of different surface treatments on bond strength between leucite reinforced feldspathic ceramic and composite resin. J Dent. 2010 Jan;38(1):39-43.

3 Ozcan M, Valandro LF, Amaral R, Leite F, Bottino MA. Bond strength durability of a resin composite on a reinforced ceramic using various repair systems. Dent Mater. 2009 Dec;25(12):1477-1483.

4 May LG, Passos SP, Capelli DB, Ozcan M, Bottino MA, Valandro LF. Effect of silica coating combined to a MDPbased primer on the resin bond to Y-TZP ceramic. Journal of Biomedical Materials Research Part B Applied Biomaterials 2010;95:69-74.

5 Passos SP, May LG, Barca DC, Ozcan M, Bottino MA, Valandro LF. Adhesive quality of self-adhesive and conventional adhesive resin cement to Y-TZP ceramic before 
and after aging conditions. Operative Dentistry 2010; 35:689-696.

6 Castro HL, Corazza PH, Paes-Ju' nior T de A, Della Bona A. Influence of Y-TZP ceramic treatment and different resin cements on bond strength to dentin. Dental Materials 2012;28:1191-1197.

7 Studart AR, Filser F, Kocher P, Gauckler LJ. In vitro life time of dental ceramics under cyclic loading in water. Biomaterials 2007;28:2695-2705.

8 Zhang Y, Lawn BR, Rekow ED, Thompson VP. Effect of sandblasting on the long-term performance of dental ceramics. Journal of Biomedical Materials Research Part B Applied Biomaterials 2004;71B:381-386.

9 Scherrer SS, Cattani-Lorente M, Vittecoq E, de Mestral F, Griggs JA, Wiskott HW. Fatigue behavior in water of YTZP zirconia ceramics after abrasion with $30 \mu \mathrm{m}$ silicacoated alumina particles. Dental Materials 2011;27:28-42.

10 Koizumi H, Nakayama D, Komine F, Blatz MB, Matsumura $\mathrm{H}$. Bonding of resin-based luting cements to zirconia with and without the use of ceramic priming agents. $J$ of Adhesive Dentistry 2012;14:385-392.

11 Takeuchi K, Fujishima A, Manabe A, Kuriyama S, Hotta Y, Tamaki Y, et al. Combination treatment of tribochemical treatment and phosphoric acid ester monomer of zirconia ceramics enhances the bonding durability of resin-based luting cements. Dental Materials J 2010;29:316-323

12 Chen L, Suh BI. Bonding of resin materials to all-ceramics: a review. Current Research in Dentistry 2012;3:7-17.

13 Chen L, Suh BI, Brown D, Chen X. Bonding investigation of primed zirconia ceramics for evidence of chemical bonding and improved bond strength. American J of Dentistry 2012;25:103-108.

14 Pashley DH, Tay FR, Breschi L, Tjaderhane L, Carvalho RM, Carrilho M, Tezvergil-Mutluay A State of the art etchand-rinse adhesives. Dent Mater 2011;7:1-16

15 Isolan CP, ValenteL, Münchow E, Basso G, Pimentel A, Schwantz J, da Silva AV and Moraes R. Bond strength of a universal bonding agent and other contemporary dental adhesives applied on enamel, dentin, composite, and porcelain. Applied adhesion science2014;2-25.

16 Kim JY., Woo JS., Lee IB., Yi YA., Hawng JY., Seo DG. Performance of universal adhesives on bonding to leucite reinforced ceramic. Biomaterials Res. 2015; 19:1-6.

17 Amaral M., Belli R., Cesar PF. , Valandro LF., Petschelt A., Lohbauer $\mathrm{U}$. The potential of novel primers and universal adhesives to bond to zirconia. J. of Dentistry. 2014;42; 90-98

18 Azimian F, Klosa K, Kern M. Evaluation of a new universal primer for ceramics and alloys. J of Adhesive Dentistry 2012;14:275-282. 